\title{
Immunomodulatory effects of inosine pranobex on cytokine production by human lymphocytes
}

\author{
WITOLD LASEK ${ }^{*}$ \\ MICHAE JANYST ${ }^{1}$ \\ RAFAŁ WOLNY ${ }^{1}$ \\ ŁUKASZ ZAPAŁA ${ }^{1}$ \\ KATARZYNA BOCIAN ${ }^{2}$ \\ NADZIEJA DRELA ${ }^{2}$ \\ ${ }^{1}$ Department of Immunology \\ Centre of Biostructure Research \\ Medical University of Warsaw \\ Warsaw, Poland \\ ${ }^{2}$ Department of Immunology \\ Faculty of Biology \\ University of Warsaw, Warsaw, Poland
}

Accepted November 20, 2014

\begin{abstract}
Inosine pranobex (inosine dimepranol acedoben, isoprinosine) (Inos) is an immunomodulatory and antiviral drug used in some viral infections, especially in patients with weakened immunity. In the present study, effects of Inos on the production of cytokines attributable to Th1 (IL-2, IFN- $\gamma$, and TNF- $\alpha$ ) or Th2 cells (IL-4, IL-5, and IL-10) were tested in human peripheral blood lymphocyte cultures stimulated with phytohemagglutinin (PHA). Inos enhanced TNF- $\alpha$ secretion significantly (in short-term - 24-hour, and prolonged term - 72-hour cultures) and IFN- $\gamma$ (in 72-hour cultures). Surprisingly, production of IL-10 by PHA-stimulated lymphocytes was suppressed by Inos in a dose-dependent manner in both 24-hour and 72-hour cultures. These results shed some light on immunomodulatory properties of Inos and suggest applicability of this agent in patients with a depressed function of the immune system.
\end{abstract}

Keywords: inosine pranobex, immunomodulation, cytokines, IL-10

Inosine pranobex (inosine dimepranol acedoben, isoprinosine) (Inos) is a synthetic agent composed of the $p$-acetamidobenzoate salt of $N, N$-dimethylamino-2-propanol and inosine at a 3:1 molar ratio (1). The drug exerts modest antiviral effects $(1,2)$, on the one hand, and immunostimulatory and immunorestorative activity $(3,4)$, on the other hand. It has been found effective in subacute sclerosing panencephalitis (5), herpes virus infections (2), and some other viral infections $(1,2)$. Treatment with Inos was also beneficial in HIV-infected patients (6). Studies in animal models showed that Inos restored defective or suppressed immunity resulting, for example, from viral infections, autoimmunity or aging $(3,4,7)$.

The most consistent effect of Inos has been enhancement of antigen- or mitogen-stimulated lymphocyte proliferation $(7,8)$. However, this agent is not itself a mitogen and, when used alone, does not stimulate blastogenesis (8). When administered in vivo, Inos has also been reported to increase the total number of $\mathrm{CD}^{+}$lymphocytes $(9,10)$, stimulate $\mathrm{NK}$ activity and augment the function of mononuclear phagocytic cells, at least in some models, culture conditions or in relation to the immunological status of the donor $(2,4,11)$.

\footnotetext{
*Correspondence; e-mail: witold.lasek@wum.edu.pl
} 
In vitro studies have revealed that Inos potentiates mitogen-induced proliferation of $\mathrm{T}$ lymphocytes $(7,8,11)$, NK cell activity (11), and stimulates production of some cytokines (12). There are suggestions that Inos augments preferentially the Th1 cell function but detailed studies have not been conducted.

The immunomodulatory effect of Inos on cells of the immune system can be partly attributed to the influence of this agent on cytokine production and secondary effects of these mediators on the cell function and activity. Inos has been shown to co-stimulate production of IL-2 in cultures of lymphocytes incubated with mitogen (12) and overproduction of this cytokine may certainly explain the increased rate of lymphocyte proliferation in the presence of Inos.

In the present study, we examined a cytokine profile (IL-2, IL-4, IL-5, IL-10, IFN- $\gamma$, and TNF- $\alpha$ ) produced by PHA-stimulated human peripheral blood lymphocytes co-incubated with different concentrations of Inos. Given that cytokines form a complex regulatory network and are produced sequentially $(13,14)$, the levels of cytokines were measured independently in one- and three-day lymphocyte cultures.

\section{EXPERIMENTAL}

\section{Preparation of cells}

Peripheral blood mononuclear cells (PBMCs) were prepared from buffy coats obtained from the Regional Centre of Blood Donation and Blood Therapy, Warsaw, Poland. The cells were isolated by the Ficoll-Hypaque (Histopaque-1077, Sigma-Aldrich, USA) density gradient sedimentation. After washing in PBS, PBMCs were counted and aliquoted in cryotubes $\left(1.8 \times 10^{7}\right.$ cells in $1.8 \mathrm{~mL}$ of $9 \%$ DMSO in culture medium). All cryotubes were then placed into a freezing container (Nalgene, Sigma-Aldrich), cooled to $-80^{\circ} \mathrm{C}$ overnight and transferred to liquid nitrogen.

\section{Inosine pranobex}

Inosine pranobex was kindly provided by Gedeon Richter Polska, Poland. Shortly before experiments, stock solutions of Inos were prepared in culture medium $\left(1 \mathrm{mg} \mathrm{mL}^{-1}\right)$, filtered (0.2- $\mu \mathrm{m}$ pore size) (Filtropur S 0.2, Sarstedt, Germany), and adequate volumes of stock solution were added to lymphocyte cultures to obtain final concentrations: 50, 100, or $200 \mathrm{mg} \mathrm{L}^{-1}$.

\section{Culture conditions and stimulation of cytokine production by lymphocytes}

After thawing and washing in PBS, cryopreserved PBMCs were re-suspended in RPMI-1640 medium supplemented with antibiotics (penicillin, $100 \mathrm{U} \mathrm{mL}^{-1}$ and streptomycin, $0.1 \mathrm{~g} \mathrm{~L}^{-1}$ ) + antimycotic (Amphotericin B, $0.25 \mathrm{mg} \mathrm{L}^{-1}$ ) (Sigma-Aldrich) and $10 \%$ heatinactivated fetal bovine serum (Gibco-Invitrogen, USA) and incubated for 1 hour in plastic tissue culture flasks [Becton Dickinson (BD) Falcon, USA] at $37{ }^{\circ} \mathrm{C}$ in a humidified atmosphere containing $5 \% \mathrm{CO}_{2}$ to deplete monocytes. 
Suspension of non-adherent cells, regarded as lymphocytes, was retrieved after gentle shaking of the flask. The lymphocytes were cultured with or without phytohemagglutinin (PHA-P, Sigma-Aldrich) (final concentration: $5 \mathrm{mg} \mathrm{L}^{-1}$ ) and/or with Inos (final concentrations: 50,100 or $\left.200 \mathrm{mg} \mathrm{L}^{-1}\right)$ in 24-well plates (BD Falcon) $\left(2 \times 10^{5}\right.$ cells in $1 \mathrm{~mL}$ ) for 24 or 72 hours. At the end of incubation period, culture supernatants were collected, centrifuged, frozen and kept at $-80{ }^{\circ} \mathrm{C}$ before being analyzed. We decided to stimulate cells with PHA instead of other stimulants (e.g., anti-CD3 monoclonal antibodies) to enable a comparison of our results with other authors.

\section{Measurement of cytokine level in culture supernatants}

Cytokine concentrations were measured in supernatants from cultured lymphocytes, isolated separately from seven buffy coats obtained from the Regional Centre of Blood Donation and Blood Therapy. To characterize Th1/Th2 cytokine profiles in culture supernatants, a BD ${ }^{\mathrm{TM}}$ Cytometric Bead Array (CBA) human Th1/Th2 cytokine kit was used (BD Biosciences, USA) according to the manufacturer's protocol. Samples were acquired using a FACSCalibur flow cytometer (Becton-Dickinson, USA). BD CellQuest Software was used to analyze the samples and data were formatted for subsequent analysis using the BD CBA Software.

\section{Flow cytometry}

Identification of T cells expressing receptors for IL-2 was performed using PE mouse anti-human CD3 and FITC mouse anti-human CD25 monoclonal antibodies (both from BD Biosciences). Lymphocytes, prepared as described above, were cultured with PHA (5 $\mathrm{mg} \mathrm{L}^{-1}$ ) and with Inos (50, 100 or $200 \mathrm{mg} \mathrm{L}^{-1}$ ) in 24-well plates for 5 days. After routine preparation and staining, the cells were analyzed using a FACScan flow cytometer (Becton-Dickinson). Expression of CD3/CD25 markers of lymphocytes was detected in four independent experiments.

\section{Statistical analysis}

Data are expressed as the mean \pm SEM of absolute values of cytokines in lymphocyte cultures for $n=7$ donors. Statistical analysis was performed using paired Student's $t$-test (two-tailed).

\section{RESULTS AND DISCUSSION}

\section{Effects of Inos on cytokine production by resting lymphocytes}

The profile of Th1- and Th2-type cytokines produced by resting (unstimulated) lymphocytes, incubated with different concentrations of Inos, was tested either in 24 or 72-hour cultures (Table I). No IL- 4 and IL-5 or trace quantities of these cytokines were produced either in control cultures (incubated without Inos) or in cultures of lymphocytes incubated with 50, 100, or $200 \mathrm{mg} \mathrm{L}^{-1}$ Inos. Very low amounts of IL-2 were found in supernatants from 24-hour lymphocyte cultures, both in controls and in cultures incubated with 
W. Lasek et al.: Immunomodulatory effects of inosine pranobex on cytokine production by human lymphocytes, Acta Pharm. 65 (2015) $171-180$.

Table I. Effect of Inos on the production of Th1- and Th2-type cytokines in cultures of resting lymphocytes (without $P H A)(n=7)$ incubated for 24 or 72 hours

\begin{tabular}{|c|c|c|c|c|c|c|c|}
\hline \multirow{3}{*}{$\begin{array}{l}\text { Time of } \\
\text { incubation } \\
\text { (h) }\end{array}$} & \multirow{3}{*}{$\begin{array}{l}\text { Concentra- } \\
\text { tion of Inos } \\
\left(\mathrm{mg} \mathrm{L}^{-1}\right)\end{array}$} & \multicolumn{6}{|c|}{ Amount of cytokines in cultures $\left(\right.$ ng L L $\left.^{-1}\right)\left(\right.$ mean range) ${ }^{\mathrm{a}}$} \\
\hline & & \multicolumn{3}{|c|}{ Th1-type cytokines } & \multicolumn{3}{|c|}{ Th2-type cytokines } \\
\hline & & IL-2 & IFN- $\gamma$ & TNF- $\alpha$ & IL-4 & IL-5 & IL-10 \\
\hline \multirow{4}{*}{24} & 0 & $5(3-9)$ & $10(2-35)$ & $233(2-424)$ & 0 & $1(0-2)$ & $172(3-418)$ \\
\hline & 50 & $6(2-11)$ & $15(0-42)$ & $251(3-457)$ & $0(0-1)$ & $1(0-2)$ & $183(5-434)$ \\
\hline & 100 & $3(0-6)$ & $6(1-17)$ & $183(3-344)$ & 0 & 0 & $143(3-410)$ \\
\hline & 200 & $3(2-5)$ & $6(0-19)$ & $203(2-359)$ & 0 & $0(0-1)$ & $137(3-359)$ \\
\hline \multirow{4}{*}{72} & 0 & $21(11-34)$ & $88(0-278)$ & $17(1-33)$ & 0 & $1(0-2)$ & $112(4-257)$ \\
\hline & 50 & $18(4-35)$ & $112(4-294)$ & $24(1-47)$ & 0 & $1(0-2)$ & $116(5-298)$ \\
\hline & 100 & $16(9-26)$ & $63(4-180)$ & $15(2-33)$ & $0(0-1)$ & $1(0-2)$ & $92(3-264)$ \\
\hline & 200 & $18(6-39)$ & $40(0-127)$ & $17(1-32)$ & $0(0-2)$ & $1(0-2)$ & $83(4-206)$ \\
\hline
\end{tabular}

${ }^{\mathrm{a}} n=7$

Inos. Low production of IL-2 was also observed upon prolonged, 72-hour incubation. No correlations between IL-2 levels and Inos concentrations were found. Higher amounts of IFN- $\gamma$ were produced in both controls and lymphocyte cultures incubated with Inos but, like in cultures assessed for IL-2, no relationships between the amounts of IFN- $\gamma$ and Inos concentrations were found. In contrast to IL- 2 and IFN- $\gamma$, significantly higher amounts of TNF- $\alpha$ were detected in supernatants from 24-hour cultures than in those from prolonged incubations. However, similarly to IL-2 and IFN- $\gamma$, large interindividual variability of the cytokine levels was observed and no clear dependence on Inos concentrations and no statistically significant differences were observed. Notably, the level of TNF- $\alpha$ production was inherent to the individual donor rather than to culture conditions and the presence of Inos during incubation.

IL-10, unlike other cytokines, was produced in comparable amounts in 24- and 72hour cultures. Similarly, however, large interindividual differences in the level of this cytokine were found. Peculiarly, some inverse trends were observed: lowest amounts of IL-10 predominated in lymphocyte cultures incubated with higher concentrations of Inos mean \pm SEM [in 24-hour cultures: controls $\left(172 \pm 65 \mathrm{ng} \mathrm{L}^{-1}\right)$ vs. $200 \mathrm{mg} \mathrm{L}^{-1}$ Inos $(137 \pm 53$ ng L $\left.{ }^{-1}\right), p=0.051$; in 72-hour cultures: 112 vs. $\left.83 \mathrm{ng} \mathrm{L}^{-1}, p=0.075\right]$.

\section{Effects of Inos on cytokine production by PHA-stimulated lymphocytes}

Production of Th1-type cytokines. - There were striking differences in the level of IL-2 in supernatants from 24- and 72-hour cultures of PHA-stimulated lymphocytes: amounts of IL-2 were more than 30 times lower upon prolonged incubation in comparison with 24hour cultures (mean 12 vs. $386 \mathrm{ng} \mathrm{L}^{-1}$ ). The level of IL-2 in 24- and 72-hour cultures did not correlate with Inos concentrations (Fig. 1a). Similarly to IL-2, the presence of Inos in 
a)

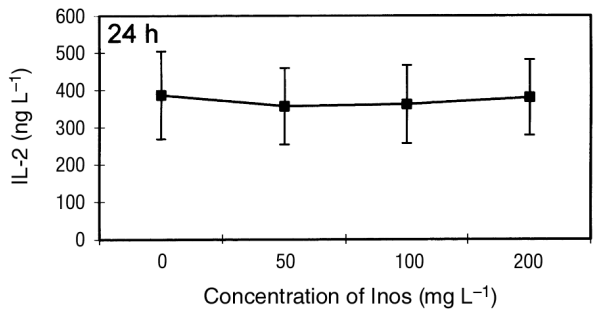

b)

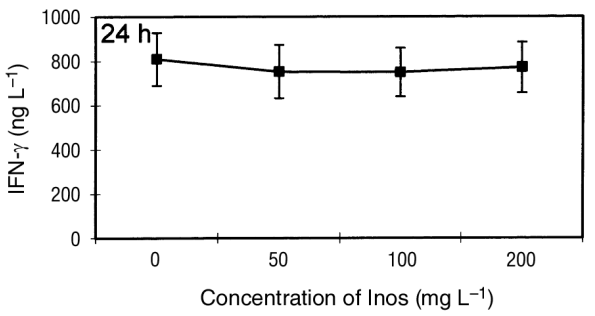

c)

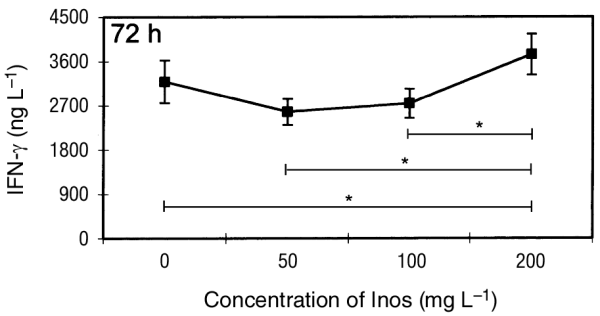

d)

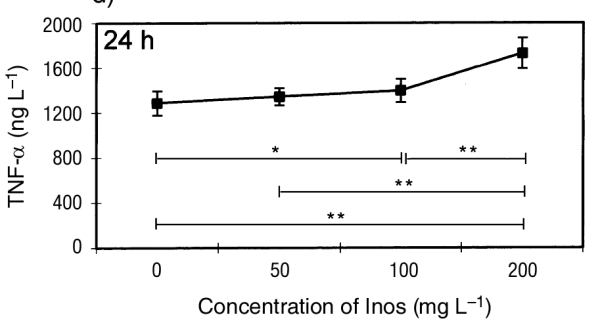

e)

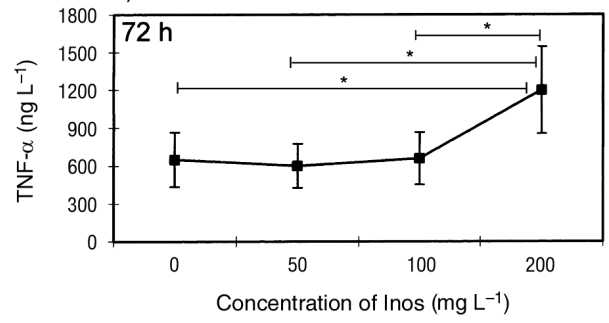

Fig. 1. Effect of Inos on production of Th1-type cytokines (IL-2, IFN- $\gamma$, and TNF- $\alpha$ ) in lymphocyte cultures stimulated with PHA for 24 hours ( $a, b$, and d), and for 72 hours (c and e). Peripheral blood lymphocytes of seven donors were incubated with $5 \mathrm{mg} \mathrm{L}^{-1} \mathrm{PHA}$ and 50, 100, or $200 \mathrm{mg} \mathrm{L}^{-1}$ Inos for 24 or 72 hours. Data are expressed as means \pm SEM. Statistically significant differences: ${ }^{*} p<0.05$, ${ }^{* *} p<0.01$.

24-hour cultures of PHA-stimulated lymphocytes did not affect the level of IFN- $\gamma$ (Fig. 1b). However, prevalence of higher amounts of this cytokine was observed in 72-hour cultures in which lymphocytes were incubated with $200 \mathrm{mg} \mathrm{L}^{-1}$ Inos (Fig. 1c). TNF- $\alpha$ is a pleiotropic cytokine produced mainly by mononuclear phagocytic cells but also by stimulated Th1 cells and NK cells $(13,15)$. In our studies, a dose-dependent relationship between the cytokine level and Inos concentration was observed in 24-hour cultures (Fig. 1d). Similarly, the highest amounts of TNF- $\alpha$ were found in supernatants from PHA-induced lymphocyte cultures incubated with the highest concentration of Inos in 72-hour cultures (185\% of controls, $p<0.05$, Fig. 1e).

The order, amount and rate of cytokine production, both in vivo and in vitro, are characteristic of each individual cytokine and result from cross-regulatory interactions be- 
tween cytokines, the strength of the stimulus and other factors $(13,16,17)$. The type of cytokines as well as the sequence of their production in our studies were similar to those reported by other authors (14).

The ability of Inos to influence production of IL-2 (and some other cytokines) was tested in ex vivo experiments in the past, in mitogen-stimulated lymphocyte cultures from animals or patients treated with this drug. Splenocytes from mice treated with Inos produced more IL-2 in comparison with the controls (12) but the effect was not observed in lymphocyte cultures in HIV-infected patients treated with Inos (18). In the latter studies, the authors also reported no effects of Inos on the production of IL-1, IL-6, and TNF- $\alpha$. In a mouse model (mitogen-stimulated splenocytes incubated with Inos), Inos did not affect the production of IL-4 and IFN- $\gamma$ in 24-hour cultures. These results differs from those obtained in our studies but may be attributed to the different sources of lymphocytes (mice vs. humans), mitogen (Con A vs. PHA), differences in assays used to measure cytokines (bioassay vs. CBA FACS), and concentrations of Inos used during incubation [lower doses of Inos were used, for example, in the study by Milano (12)]. Notably, the activity of IL-2 and IFN- $\gamma$ was higher, and the IL-4 level was lower in mitogen-driven cultures from mice treated with Inos. The authors suggested preferential promotion of the Th1-type response by Inos (12). Our results are consistent, in part, with this suggestion.

In a recent paper by Petrova et al. (19), the effect of treatment with Inos on serum cytokine level was examined in ten healthy adults. Increased levels of IL-2, IL-10 and TNF- $\alpha$ were observed following Inos administration. However, methodological shortcomings (lack of controls) and ambiguous interpretations of some results make this study difficult to comment.

Production of Th2-type cytokines. - IL-4, the canonical Th2-type cytokine, was produced in small amounts in 24-hour cultures of PHA-stimulated lymphocytes and in negligible amounts (mostly less than $10 \mathrm{ng} \mathrm{L}^{-1}$ ) following prolonged incubation. In 24-hour cultures, Inos seemed to co-stimulate IL-4 production in a dose-dependent manner (mean amounts for $0,50,100$, and $200 \mathrm{mg} \mathrm{L}^{-1}$ concentrations of Inos were 24.7, 25.1, 25.7, and $27.7 \mathrm{ng} \mathrm{L}^{-1} \mathrm{IL}-4$, respectively) (Fig. 2a). However, due to high interindividual variability, no statistically significant differences were found. Similarly, although the highest levels of IL-5 were consistently demonstrated when PHA-stimulated cells were co-cultured with the highest concentrations of Inos, the differences between Inos and the controls did not reach statistical significance (Figs. $2 b$ and c). In comparison with IL-4 and IL-5, levels of IL-10 in PHAstimulated cultures of lymphocytes were, generally, much higher (about 20 times). Moreover, a quite different effect of Inos on IL-10 production was observed: the negative relationship between Inos concentration and the amount of IL-10 in the culture supernatant was obvious. In 24-hour cultures, for example, mean values for 0, 50, 100, and $200 \mathrm{mg} \mathrm{L}^{-1}$ Inos were $629,601,549$, and $497 \mathrm{ng} \mathrm{L}^{-1}$, respectively (Fig. 2d). Lower amounts of IL-10 prevailed also in supernatants from cultures of lymphocytes co-incubated with Inos for 72 hours but a statistically significant difference, compared to the controls, was observed only for $50 \mathrm{ng} \mathrm{L}^{-1}$ Inos concentration (Fig. 2e).

The dose-dependent decrease of IL-10 production in cultures with Inos, in contrast to more or less co-stimulatory effects of Inos on the production of other cytokines, does not seem to be accidental: the same pattern of response (the higher the concentration of Inos, the lower the amounts of IL-10 in cultures) was shown in all but one donor, in supernatants collected in three independent experiments. IL-10 is a broadly expressed cytokine playing 
a)

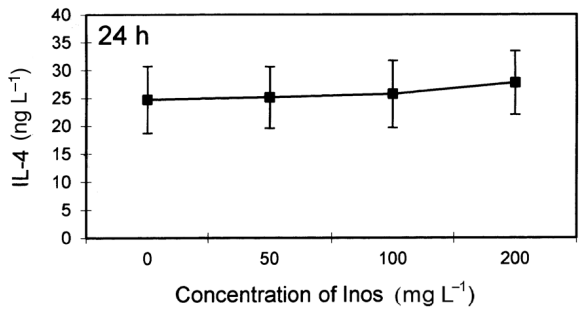

b)

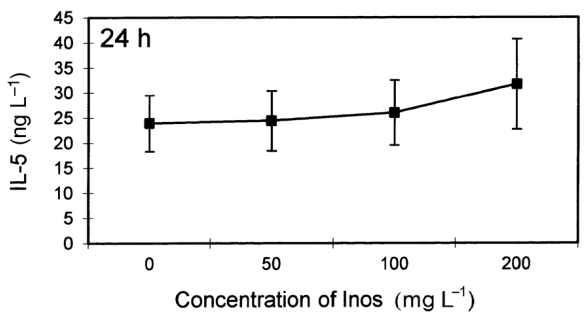

c)

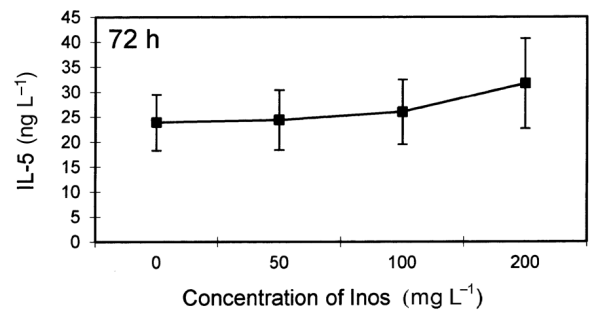

d)

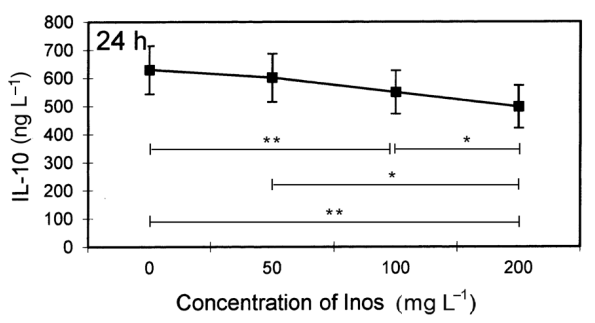

e)

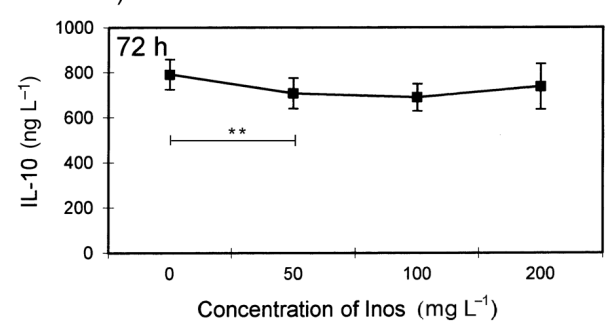

Fig. 2. Effect of Inos on production of Th2-type cytokines (IL-4, IL-5, and IL-10) in lymphocyte cultures stimulated with PHA for 24 hours (a, b, and d), and for 72 hours (c and e). Peripheral blood lymphocytes of seven donors were incubated with $5 \mathrm{mg} \mathrm{L}^{-1} \mathrm{PHA}$ and 50, 100, or $200 \mathrm{mg} \mathrm{L}^{-1}$ Inos for 24 or 72 hours. Data are expressed as means \pm SEM. Statistically significant differences: ${ }^{*} p<0.05$, ${ }^{* *} p<0.01$.

a dominant role in suppression of innate and adaptive immunity (20) and is produced mainly by monocytes and macrophages but also by lymphoid cells such as Th2, T $\gamma \delta$ cells, and B lymphocytes (14, 20-23), and, under certain conditions, by Th1 cells (22, 23). It belongs classically to the group of Th2-type cytokines but in humans it is also produced in large amounts by a subset of regulatory T cells - Tr1 lymphocytes $(20,24)$. Although it cannot be excluded that small quantities of IL-10 in supernatants in our experiments derive from residual phagocytic cells (monocytes), the strong dependence of IL-10 production on the presence of PHA in cultures clearly shows that the primary source of this cytokine are lymphocytes. We cannot offer an unequivocal explanation of lower levels of IL-10 detected in cultures incubated with Inos but, since IL-4 and IFN- $\gamma$ have been reported to inhibit production of this cytokine $(20,22)$, it may be that the suppression of IL-10 production is, 

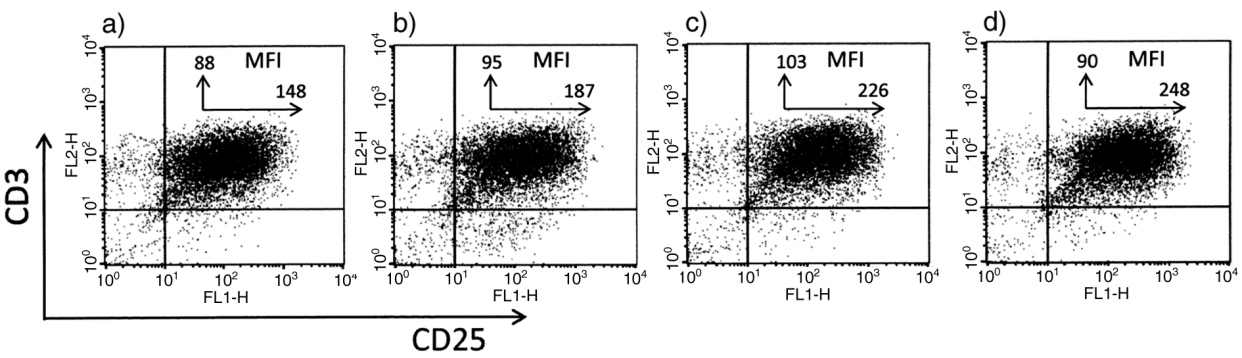

Fig. 3. Effect of Inos on CD25 marker (IL-2R $\alpha$ ) of T lymphocytes (CD3-positive). Peripheral blood lymphocytes incubated with $5 \mathrm{mg} \mathrm{L}^{-1}$ PHA and: a) 0, b) 50, c) 100, or d) $200 \mathrm{mg} \mathrm{L}^{-1}$ Inos for 5 days. Representative results of one of four experiments are shown. Mean fluorescence intensity (MFI) measured in flow cytometer is shown for both CD3 and CD25 marker.

at least in part, secondary to the increased synthesis of IL-4 and IFN- $\gamma$ (and perhaps other cytokines). One can also hypothesize that Inos preferentially inhibits the development and/or function of $\operatorname{Tr} 1$ cells.

Expression of CD25 molecule. - To shed some light on the reason for the low amounts of IL-2 in supernatants from cultures of lymphocytes incubated for a prolonged time and to explain no effect of Inos on the IL-2 level in 24-hour cultures, we performed analyses of CD25 marker expression ( $\alpha$-chain of IL-2R) on T lymphocytes. As shown in Fig. 3, incubation of PHA-stimulated lymphocytes with different concentrations of Inos led to a dosedependent increase in IL-2R expression. One may hypothesize that prolonged incubation of lymphocytes results in immediate binding of IL-2 to IL-2R and utilization of this cytokine in quickly proliferating cells in an autocrine and paracrine manner. The dose-dependent relationship was confirmed in 3 of the 4 independent experiments.

\section{CONCLUSIONS}

The present paper clearly shows modulatory effects of Inos on cytokine production in PHA-stimulated lymphocyte cultures and provides, for the first time, the evidence that Inos does not only stimulate effector functions of lymphocytes but can also suppress regulatory (inhibitory) mechanisms. Since the immune dysfunction is often associated with some viral infections $(25,26)$, our studies show that application of Inos could be beneficial, as an adjuvant therapy, in selected groups of patients, e.g., those suffering from recurrent viral infections.

Abbreviations. - BD - Becton Dickinson, DMSO - dimethyl sulfoxide, IFN - interferon, IL - interleukin, Inos - inosine pranobex, NK - natural killer, PBMCs - peripheral blood mononuclear cells, PHA - phytohemagglutinin, TNF - tumor necrosis factor.

Acknowledgements. - This work was supported by Gedeon Richter Poland, Grant UK1/283. Buffy coats are recognized in Poland as waste product in the process of blood preparation, so no approval by Ethical Committee was necessary to carry out research described in the paper. 


\section{REFERENCES}

1. D. M. Campoli-Richards, E. M. Sorkin and R. C. Heel, Inosine pranobex. A preliminary review of its pharmacodynamic and pharmacokinetic properties, and therapeutic efficacy, Drugs 32 (1986) 383-424.

2. H. Ohnishi, H. Kosuzume, H. Inaba, M. Ohkura, S. Shimada and Y. Suzuki, The immunomodulatory action of inosiplex in relation to its effects in experimental viral infections, Int. J. Immunopharmacol. 5 (1983) 181-196.

3. H. Ohnishi, H. Kosuzume, H. Inaba, M. Okura, Y. Morita, H. Mochizuki and Y. Suzuki, Mechanism of host defence suppression induced by viral infection: mode of action of inosiplex as an antiviral agent, Infect. Immun. 38 (1982) 243-250.

4. K. Y. Tsang, H. H. Fudenberg and M. J. Gnagy, Restoration of immune response of aging hamsters by treatment with isoprinosine, J. Clin. Invest. 71 (1983) 1750-1755; DOI: 10.1A2/JCI110930.

5. J. Gutierrez, R. S. Issacson and B. S. Koppel, Subacute sclerosing panencephalitis: an update, Dev. Med. Child. Neurol. 52 (2010) 901-907; DOI: 10.1111/j.1469-8749.2010.03717.x.

6. C. Pedersen, E. Sandström, C. S. Petersen, G. Norkrans, J. Gerstoft, A. Karlsson, K. C. Christensen, C. Håkanson, P. Pehrson, J. O. Nielsen, H. J. Jürgensen, and Scandinavian Isoprinosine Study Group, The efficacy of inosine pranobex in preventing the acquired immunodeficiency syndrome in patients with human immunodeficiency virus infection, New Engl. J. Med. 322 (1990) 1757-1763; DOI: 10.1056/NEJM199006213222501.

7. T. Nakamura, N. Miyasaka, R. M. Pope, N. Talal and I. J. Russell, Immunomodulation by isoprinosine: effects on in vitro immune functions of lymphocytes from humans with autoimmune diseases, Clin. Exp. Immunol. 52 (1983) 67-74.

8. J. W. Hadden, E. M. Hadden and R. G. Coffey, Isoprinosine augmentation of phytohemagglutinininduced lymphocyte proliferation, Infect. Immun. 13 (1976) 382-387.

9. J. Litzman, J. Lokaj, M. Krejci, S. Pesak and G. Morgan, Isoprinosine does not protect against frequent respiratory tract infections in childhood, Eur. J. Pediatr. 158 (1999) 32-37.

10. M. Gołębiowska-Wawrzyniak, K. Markiewicz, A. Kozar, P. Derentowicz, J. Czerwińska-Kartowicz, K. Jastrzębska-Janas, J. Wacławek, Z. M. Wawrzyniak and H. Siwinska-Gołębiowska, Immunological and clinical study on the therapeutic efficacy of inosine pranobex, Pol. Merkur. Lekarski 19 (2005) 379-382.

11. P. Hersey and A. Edwards, Effect of isoprinosine on natural killer cell activity of blood mononuclear cells in vitro and in vivo, Int. J. Immunopharmacol. 6 (1984) 315-320; DOI: 10.1016/01920561(84)90048-1.

12. S. Milano, M. Dieli, S. Millott, M. D. Miceli, E. Maltese and E. Cillari, Effect of isoprinosine on IL-2, IFN- $\gamma$ and IL-4 production in vivo and in vitro, Int. J. Immunopharmacol. 13 (1991) 1013-1018; DOI: 10.1016/0192-0561(91)90055-C.

13. S. Jiang and C. Dong, A complex issue on CD4+ T-cell subsets, Immunol. Rev. 252 (2013) 5-11; DOI: 10.1111/imr.12041.

14. S. McHugh, J. Deighton, I. Rifkin and P. Ewan, Kinetics and functional implications of Th1 and Th2 cytokine production following activation of peripheral blood mononuclear cells in primary culture, Eur. J. Immunol. 26 (1996) 1260-1265; DOI: 10.1002/eji.1830260612.

15. B. Perussia, The cytokine profile of resting and activated NK cells, Methods 9 (1996) 370-378; DOI: 10.1084/jcm.20032058.

16. M. Akdis, J. Verhagen, A. Taylor, F. Karamloo, C. Karagiannidis, R. Crameri, S. Thunberg, G. Deniz, R. Valenta, H. Fiebig, C. Kegel, R. Disch, C. B. Schmidt-Weber, K. Blaser and C. A. Akdis, Immune response in healthy and allergic individuals are characterized by a fine balance between allergen-specific T regulatory 1 and T helper 2 cells, J. Exp. Med. 199 (2004) 1567-1575. 
17. I. Striz, E. Brabcova, L. Kolesar and A. Sekerkova, Cytokine networking of innate immunity cells: a potential target of therapy, Clin. Sci. 126 (2014) 593-612; DOI: 10.1042/CS20130497.

18. B. K. Pedersen, N. Tvede, M. Diamant, J. Gerstoft, M. Bagge Hansen, P. M. Haahr, M. Hording, M. Kappel, M. Klokker, B. Soeberg and P. Skinhoj, Effects of isoprinosine treatment of HIV-positive patients on blood mononuclear cell subsets, NK- and T-cell function, tumor necrosis factor, and interleukins 1, 2, and 6, Scand. J. Immunol. 32 (1990) 641-649.

19. M. Petrova, D. Jelev and Z. Krastev, Isoprinosine affects serum cytokine levels in healthy adults, J. Interferon Cytokine Res. 30 (2010) 223-227; DOI: 10.1089/jir.2009.0057.

20. R. Sabat, G. Grutz, K. Warszawska, S. Kirsch, E. Witte, K. Wolk and J. Geginat, Biology of interleukin-10, Cytokine Growth Factor Rev. 21 (2010) 331-344; DOI: 1016/j.cytogfr.2010.09.002.

21. A. Langkjaer, B. Kristensen, B. E. Hansen, H. Schultz, L. Hegedus and C. H. Nielsen, B-cell exposure to self-antigen induces IL-10 producing B cells as well as IL-6- and TNF- $\alpha$-producing B-cell subsets in healthy humans, Clin. Immunol. 145 (2012) 1-10; DOI: 10.1016/j.clim.2012.07.004.

22. M. Saraiva and A. O'Garra, The regulation of IL-10 production by immune cells, Nat. Rev. Immunol. 10 (2010) 170-181; DOI: 10.103/nri2711.

23. G. Del Prete, M. De Carli, F. Almerigogna, M. G. Giudizi, R. Biagotti and S. Romagnani, Human IL-10 is produced by both type 1 helper (Th1) and type 2 helper (Th2) T cell clones and inhibits their antigen-specific proliferation an cytokine production, J. Immunol. 150 (1993) 353-360.

24. S. Gregori, K. S. Goudy and M. G. Roncarolo, The cellular and molecular mechanisms of immunosuppression by human type 1 regulatory T cells, Front. Immunol. 3 (2012), article 30; DOI: 10.3389/ fimmu.2012.00030.

25. A. A. Navarini, M. Recher, K. S. Lang, P. Georgiev, S. Meury, A. Bergthaler, L. Flatz, J. Bille, R. Landmann, B. Odermatt, H. Hengartner and R. M. Zinkernagel, Increased susceptibility to bacterial superinfection as a consequence of innate antiviral responses, PNAS 103 (2006) 15335-15539; DOI: $10.1073 /$ pnas.0607225103.

26. W. W. Metzger and K. Sun, Immune dysfunction and bacterial coinfections following influenza, J. Immunol. 191 (2013) 2047-2052; DOI: 10.4049/jimmunol.1301152. 